Caracterização da sífilis gestacional em um munícipio prioritário do projeto "sífilis não" Characterization of gestational syphilis in a priority municipality of the 'no syphilis" project Caracterização de la sífilis gestacional en un municipio prioritario del proyecto "no sífilis"

Recebido: 19/10/2020 | Revisado: 21/10/2020 | Aceito: 22/10/2020 | Publicado: 24/10/2020

\title{
Francisca Flaviana Alves Sobrinho
}

ORCID: https://orcid.org/0000-0001-7794-0189

Centro Universitário Santo Agostinho, Brasil

E-mail: flavianaalves8956@gmail.com

Sara Maria Marques Lima

ORCID: https://orcid.org/0000-0002-0802-0837

Centro Universitário Santo Agostinho, Brasil

E-mail: sara_maria.320@hotmail.com

Tatiana Maria Melo Guimarães

ORCID: https://orcid.org/0000-0002-2748-6771

Centro Universitário Santo Agostinho, Brasil

E-mail: tatianacorenpi@gmail.com

\section{Resumo}

A sífilis é uma doença infecciosa sistêmica de evolução crônica que pode ser transmitida principalmente por via sexual, mas também por via sanguínea e transplacentária. O Ministério da Saúde instituiu o projeto "Sífilis Não" tem como finalidade conscientizar as gestantes e suas parcerias sexuais, indivíduos sexualmente ativos e profissionais de saúde sobre formas de combate e tratamento da sífilis. O objetivo desse estudo é analisar a prevalência de sífilis na gestação na cidade de Teresina-PI de 2015 até 2019. Realizou-se uma pesquisa com abordagem quantitativa de corte transversal. No estudo caracterizou-se o perfil epidemiológico das gestantes quanto a faixa etária, escolaridade, caso de sífilis congênita de acordo com o pré-natal realizado, tratamento do parceiro realizado, idade gestacional e esquema de tratamento prescrito de acordo com dados obtidos na plataforma DATASUS. Os resultados do presente estudo mostraram um número crescente de casos ao longo dos anos, sendo que a quantidade de mulheres com pré-natal realizado foi elevado, entretanto, o estudo aponta índices muito alto de gestantes que com diagnósticos de sífilis a partir do terceiro 
trimestre de gravidez o que nos indica um início de pré-natal tardio ou uma infecção tardia. $\mathrm{O}$ maior número de casos de sífilis se deu em mulheres com baixa escolaridade e faixa etária entre 20 a 29 anos em decorrência da grande falta de informações. Diante disso, percebe-se que são necessárias mais ações preventivas contra sífilis direcionada para essa parte da população que tem dificuldade ao acesso a informações.

Palavras-chave: Sífilis; Gestantes; Doenças sexualmente transmissíveis; Cuidado pré-natal.

\begin{abstract}
The aim of this study is to analyze the prevalence of syphilis in pregnancy in the city of Teresina-PI from 2015 to 2019. An integrative literature review study was conducted using searches on the VHL, PUBMED and Google Scholar data platform, to build the article and the epidemiological profile of pregnant women was characterized in terms of age, education, case of congenital syphilis according to the prenatal care performed, treatment of the partner performed, gestational age and treatment schedule prescribed according to data obtained on the DATASUS platform. The results of the present study showed an increasing number of cases over the years, and the number of women with prenatal care performed was high, however, the study points out very high rates of pregnant women with diagnoses of syphilis from the third trimester pregnancy, which indicates a late start of prenatal care. The largest number of cases of syphilis occurred in women with low education and aged between 20 and 29 years due to the great lack of information. In view of this, it is clear that more preventive actions against syphilis are needed for this part of the population that has difficulty accessing information.
\end{abstract}

Keywords: Syphilis; Pregnant women; Sexually transmitted diseases; Prenatal care.

\title{
Resumen
}

El objetivo de este estudio es analizar la prevalencia de sífilis en el embarazo en la ciudad de Teresina-PI de 2015 a 2019. Se realizó un estudio de revisión integradora de la literatura mediante búsquedas en la plataforma de datos BVS, PUBMED y Google Scholar, para construir la Se caracterizó el artículo y el perfil epidemiológico de la gestante en cuanto a edad, escolaridad, caso de sífilis congénita según la atención prenatal realizada, tratamiento de la pareja realizado, edad gestacional y esquema de tratamiento prescrito según datos obtenidos en la plataforma DATASUS. Los resultados del presente estudio mostraron un número creciente de casos a lo largo de los años, y la cantidad de mujeres con atención prenatal realizada fue alta, sin embargo, el estudio señala tasas muy altas de mujeres embarazadas con 
diagnósticos de sífilis a partir del tercer trimestre. embarazo, lo que indica un inicio tardío de la atención prenatal. El mayor número de casos de sífilis se presentó en mujeres con bajo nivel educativo y con edades comprendidas entre los 20 y los 29 años debido a la gran falta de información. Ante esto, es evidente que se necesitan más acciones preventivas contra la sífilis para esta parte de la población que tiene dificultades para acceder a la información.

Palabras clave: Sífilis; Mujeres embarazadas; Enfermedades sexualmente transmisibles; Cuidado prenatal.

\section{Introdução}

A sífilis é uma doença infecciosa sistêmica de evolução crônica, causada pela bactéria Treponema pallidum, um organismo espiralado e delicado, podendo ser transmitido sexualmente, via transplacentária, via canal de parto e por contato direto com o sangue. Ela se caracteriza por ter vários estágios clínicos e também pode se desenvolver em períodos de latência quando não tratada (Delben \& Rodrigues, 2018).

Tornou-se conhecida na Europa no final do século XV, chamando atenção pela primeira vez durante o cerco que os franceses fizeram na cidade de Nápoles em 1945. Devido a esse episódio nasceu-se o nome de "Mal de Nápoles" ou Mal Italiano. Logo tornou- se uma pandemia devida a sua rápida disseminação e atualmente continua sendo um problema de saúde pública, pois tem impacto direto na saúde da mulher, do parceiro (a) e da criança. (RosVivancos, 2018).

Entre 1891 e 1948 foi realizado um estudo de Oslo, no qual três professores observaram 2000 pacientes sem qualquer interferência e com esse estudo, obtiveram a história natural da doença que mostra o crescimento da patologia que se alterna entre períodos de atividade com características clínicas, imunológicas e fases distintas (sífilis primária, secundária e terciária) e períodos de latência (sífilis latente). A sífilis divide-se ainda em sífilis recente, nos casos em que o diagnóstico é feito em até um ano depois da infecção, e sífilis tardia, quando o diagnóstico é realizado após um ano. Em algumas situações (seja na sífilis recente ou na tardia) a infecção pode evoluir para a neuro sífilis (D’Oliveira, 2019).

Na fase primária após a infecção, ocorre um período de incubação entre 10 a 90 dias. O primeiro sintoma é o aparecimento de uma lesão única denominada cancro duro é indolor, aparece primeiramente uma pápula de cor rósea, que progride para um vermelho mais vivo e exulceração. Na fase secundaria as lesões apresentam-se como uma erupção pouco visível de cor eritematosa (rosa sifilítica) de duração efêmera, principalmente no tronco e raiz dos 
membros, nessa fase são comuns as placas mucosas, assim como as lesões acinzentadas. As lesões cutâneas progridem para lesões mais evidentes, populosas eritemas acastanhados, sendo frequentes nos genitais. $\mathrm{Na}$ fase terciaria a inflamação nesse estágio promove destruição tecidual podendo haver comprometimento do sistema nervoso central, do sistema cardiovascular com inflamação da aorta, lesões na pele e nos ossos (Torres et al, 2019).

Grande parte da população infectada desconhece que possui a doença, devido a muitas vezes a infecção ser assintomática, ou seja, a infecção não exibe sintomas e quando se torna sintomática os portadores ignoram os sinais e sintomas e podem, sem saber, transmitir a infecção. Na gestação a sífilis pode provocar abortamento, natimortalidade, prematuridade, má formação congênita, e morte do recém-nascido (Guerra, 2017).

No Brasil, os casos de sífilis está presente em todos os estados e de acordo com a Secretária de Vigilância em Saúde (2019), no intervalo de 2010 a 2018 houve uma evolução das taxas de sífilis. Nesse período, observou-se o aumento na taxa de sífilis congênita que evoluiu de 2,4 para 9,0 de casos por mil nascidos vivos. Nos casos de sífilis adquirida houve um aumento de 34,1 casos/100.000 habitantes para 75,8 casos/100.000 habitantes. E em relação a sífilis gestacional foi relatado o aumento de $25,7 \%$.

O projeto "Sífilis Não" é uma iniciativa do Ministério da Saúde elaborado pela UFRN e concretizado pela FUNPEC, visando a ampliação de pesquisas, ações educativas e comunicações sobre formas de combate e tratamento da sífilis. A ação tem como principal finalidade conscientizar as gestantes e suas parcerias sexuais, indivíduos sexualmente ativos e profissionais de saúde (Funpec, 2018).

A campanha enfatiza de forma mais completa e dinâmica sobre a prevenção, diagnóstico e tratamento da infecção. Além de vídeos disponíveis para a população, ela conta com perfis em rede sociais que difundem informações sobre a temática da sífilis, esclarecendo dúvidas e interagindo com os internautas, ressaltando que a melhor maneira de promover a saúde e evitar doenças é por meio da comunicação e informação (Brasil, 2019).

O presente estudo se justifica devido a importância de conscientizar a população de maneira geral sobre a prevenção e tratamento da sífilis. Além de proporcionar informações sobre os casos, mostrará a importância de seu descobrimento precoce e tratamento. A sífilis gestacional é um agravo de notificação compulsória, com altos índices de casos notificados, o que permite uma reflexão para com este agravo, uma vez que possibilita a caracterização do perfil epidemiológico das mulheres durante o pré-natal e parto. Diante disso, a pesquisa tem como problema de pesquisa a seguinte: foi lançado um projeto do governo federal onde 100 municípios tornam-se prioritários no combate a sífilis, no qual Teresina-PI encontra-se na $8^{\circ}$ 
lugar de maior incidência de casos notificados. O trabalho irá enfatizar os casos de sífilis gestacional no município de Teresina no período de 2015 a 2019.

Este artigo tem como objetivo geral analisar o perfil epidemiológico dos casos notificados de sífilis em gestantes, em Teresina-PI nos anos de 2015 a 2019; e específicos: caracterizar o perfil epidemiológico das gestantes quanto a faixa etária, escolaridade, casos de sífilis congênita de acordo com o pré-natal realizado, tratamento do parceiro realizado, idade gestacional e esquema de tratamento prescrito e determinar o número de casos diagnosticados de sífilis nos anos de 2015 a 2019.

\section{Metodologia}

Trata-se de uma pesquisa com abordagem quantitativa de corte transversal. A pesquisa quantitativa é comumente usada nas ciências naturais e sociais. O método se apoia predominantemente na investigação de dados estatísticos, visando gerar medidas precisas e confiáveis que permitam uma análise estatística na tentativa de garantir precisão dos resultados que por sua vez geralmente são lógicos, estatísticos e imparciais (Patias \& Hohendorff, 2019).

Devido ser uma pesquisa quantitativa, os resultados podem ser quantificados. A pesquisa recorre linguagem matemática onde faz se a coleta de dados quantitativos ou numéricos através do uso de medições de grandeza e conseguem se por meio da metrologia. Centrada na objetividade, a pesquisa foca na análise de dados brutos (Pereira A.S. et al.2018).

O local de estudo foi o município de Teresina-PI em função das seguintes características: capital do estado do Piauí, onde a população estimada é de 864.845 (IBGE,2019); encontra-se na $8^{\circ}$ posição do ranking no projeto interfedarativo "Sífilis Não". Teresina possui uma ampla rede de prestação de serviços de saúde como hospitais, unidades básicas de saúde, policlínicas, clinicas pertencentes ao Estado ao Município, e a iniciativa privada, tornando a capital um importante centro de atendimento médico para diversas especialidades.

A coleta de dados foi realizada por meio de relatórios de dados estatísticos disponibilizados pelo governo através do Portal da Saúde (DATASUS). A análise foi feita a partir do ano de 2015 a 2019. Por se tratar de uma pesquisa onde apenas utilizou-se a análise de dados públicos do sistema de saúde, a pesquisa não necessita ser submetida ao comitê de ética. 
Os dados analisados foram extraídos da base de dados do sistema de informações públicas do DATASUS, que é o departamento de informática do Sistema Único de Saúde do Brasil. Sendo assim, a coleta foi feita por meio do TABNET, programa que disponibiliza os dados em tabelas produzidas pelo DATASUS-Ministério da Saúde. No DATASUS buscou-se as informações de Teresina-PI, sobre o número de casos notificados de sífilis em gestantes no referido município no período de 2015 a 2019, foram analisados também a faixa etária dessas gestantes, sua escolaridade, idade gestacional, a realização ou não do pré-natal, tratamento prescrito e o tratamento do parceiro. A partir das informações extraídas, foi construído tabelas, contendo o total de casos notificados de sífilis em gestantes no período já citado acima, e feito a categorização do perfil epidemiológico dessas gestantes baseados na faixa etária, escolaridade, quantidade de gestantes que realizaram pré-natal e a quantidade de gestantes que não efetuaram o pré-natal, utilizando planilhas do Microsoft Excel e Microsoft Office.

\section{Resultados}

A partir da análise dos documentos encontrados, as informações foram agrupadas e separadas em tabelas para melhor entendimento e compreensão.

A Tabela 1 retrata se houve a realização do pré-natal, em qual idade gestacional a gestante estava quando descobriu a infecção e faixa etária em que a gestante se encontrava. 
Tabela 1. Distribuição dos casos de gestantes com sífilis segundo pré-natal realizado, idade gestacional e faixa etária da gestante. Teresina-PI, 2020.

\begin{tabular}{|c|c|c|c|c|c|c|c|}
\hline Variáveis & 2015 & 2016 & 2017 & 2018 & 2019 & Total & $\%$ \\
\hline \multicolumn{8}{|l|}{ Pré-natal } \\
\hline Pré-natal realizado & 174 & 170 & 167 & 172 & 67 & 750 & $79,96 \%$ \\
\hline Pré-natal não realizado & 49 & 33 & 36 & 50 & 8 & 176 & $18,76 \%$ \\
\hline $\begin{array}{l}\text { Pré-natal Ignorado } \\
\text { Idade Gestacional }\end{array}$ & 1 & 1 & 2 & 3 & 5 & 12 & $1,28 \%$ \\
\hline $\begin{array}{l}\text { Idade gestacional }\left(1^{\circ}\right. \\
\text { trimestre) }\end{array}$ & 30 & 41 & 43 & 61 & 29 & 204 & $21,8 \%$ \\
\hline $\begin{array}{l}\text { Idade gestacional }\left(2^{\circ}\right. \\
\text { trimestre) }\end{array}$ & 43 & 57 & 56 & 68 & 40 & 264 & $28,3 \%$ \\
\hline $\begin{array}{l}\text { Idade gestacional ( } 3^{\circ} \\
\text { trimestre) }\end{array}$ & 52 & 39 & 76 & 202 & 52 & 421 & $45,1 \%$ \\
\hline $\begin{array}{l}\text { Idade gestacional } \\
\text { ignorada }\end{array}$ & 2 & 2 & 8 & 15 & 14 & 41 & $4,3 \%$ \\
\hline \multicolumn{8}{|l|}{$\begin{array}{l}\text { Faixa Etária da } \\
\text { gestante }\end{array}$} \\
\hline $\begin{array}{l}\text { Gestantes com idade } \\
\text { entre } 10 \text { e } 14 \text { anos }\end{array}$ & 1 & 2 & 1 & 3 & 0 & 7 & $0,7 \%$ \\
\hline $\begin{array}{l}\text { Gestantes com idade } \\
\text { entre } 15 \text { e } 19 \text { anos }\end{array}$ & 34 & 39 & 39 & 82 & 19 & 214 & $23,2 \%$ \\
\hline $\begin{array}{l}\text { Gestantes com idade } \\
\text { entre } 20 \text { e } 29 \text { anos }\end{array}$ & 60 & 65 & 97 & 182 & 75 & 479 & $52,3 \%$ \\
\hline $\begin{array}{l}\text { Gestantes com idade } \\
\text { entre } 30 \text { e } 39 \text { anos }\end{array}$ & 30 & 31 & 40 & 68 & 26 & 195 & $21,3 \%$ \\
\hline $\begin{array}{l}\text { Gestantes com idade } \\
\text { igual ou superior a } 40 \\
\text { anos }\end{array}$ & 2 & 2 & 6 & 11 & 4 & 25 & $2,7 \%$ \\
\hline
\end{tabular}

Fonte: Pesquisa direta em base de dados. Teresina (2020).

Foi observado que 79,96\% referiram ter realizado pré-natal, 18,76\% referiram não ter realizado pré-natal, e 1,28\% ignoraram o questionamento. Mostra também casos de mulheres gestantes portadoras da sífilis diagnosticadas e notificadas na cidade de Teresina-PI no ano de 2015 até o ano de 2019 de acordo com a idade gestacional. Mostrou-se que 21,8\% das mães descobriram a patologia no primeiro trimestre da gestação, $28,3 \%$ no $2^{\circ}$ semestre de gestação, 45,1\% descobriram no $3^{\circ}$ semestre de gestação, 4,3\% não informaram a idade gestacional. No que se refere a esquema de tratamento, 91,8\% informaram que fizeram o uso de Penicilina, $7,1 \%$ informaram que utilizaram outro esquema de tratamento, $1,5 \%$ das gestantes não realizaram nenhum tratamento e 0,9\% ignoraram o questionamento. Em relação à faixa etária da gestante, onde foi notificado que 1,1\% tinha de 10 a 14 anos, $25 \%$ de 15 a 19 anos, $52 \%$ de 20 a 29 anos, $19,7 \%$ de 30 a 39 anos, 2,3\% relatou ter 40 anos ou mais e $0 \%$ ignoraram o questionamento. A tabela relata sobre o tratamento do parceiro, onde foi observado que $40,9 \%$ realizou o tratamento, $53,8 \%$ não realizou tratamento e apenas $5,2 \%$ ignoraram o questionamento. 
Research, Society and Development, v. 9, n. 10, e8889109414, 2020

(CC BY 4.0) | ISSN 2525-3409 | DOI: http://dx.doi.org/10.33448/rsd-v9i10.9414

A Tabela 2 retrata sobre a escolaridade das gestantes, onde foi averiguado o nível escolar de cada uma das pacientes.

Tabela 2. Distribuição dos casos de gestantes com sífilis quanto à escolaridade.

\begin{tabular}{|c|c|c|c|c|c|c|c|}
\hline Varíáveis & 2015 & 2016 & 2017 & 2018 & 2019 & Total & $\%$ \\
\hline \multicolumn{8}{|l|}{ Escolaridade } \\
\hline Analfabeto & 1 & 1 & - & 2 & 1 & 5 & $0,59 \%$ \\
\hline $1^{2}$ a $4^{2}$ série incom pleta & 14 & 6 & 10 & 13 & 4 & 47 & $5,57 \%$ \\
\hline $4^{2}$ série incompleta & 4 & 4 & 9 & 6 & 2 & 25 & $2,94 \%$ \\
\hline $5^{2}$ a $8^{2}$ série incom pleta & 32 & 37 & 36 & 76 & 31 & 212 & $16,71 \%$ \\
\hline Fundam ental com pleto & 10 & 10 & 14 & 30 & 11 & 75 & $8,82 \%$ \\
\hline Médio incom pleto & 33 & 30 & 39 & 67 & 23 & 192 & $22,59 \%$ \\
\hline Médio completo & 17 & 25 & 45 & 89 & 29 & 205 & $24,12 \%$ \\
\hline Superior incom pleto & 2 & 3 & 3 & 12 & 3 & 23 & $0,94 \%$ \\
\hline Nầo se aplica & - & - & - & $\cdot$ & 1 & 1 & $0,12 \%$ \\
\hline Ignora do & 13 & 22 & 27 & 47 & 18 & 157 & $19,94 \%$ \\
\hline
\end{tabular}

Fonte: Pesquisa direta em base de dados. Teresina (2020).

A Tabela mostra que $5,57 \%$ declararam da $1^{\circ}$ a $4^{\circ}$ série incompleta, $2,94 \%$ até a $4^{\circ}$ série completa, $16,71 \%$ dá $5^{\circ}$ a $8^{\circ}$ série incompleta, $8,82 \%$ declararam o fundamento completo, 22,59\% médio incompleto, $24,12,5 \%$ médio completo, $0,94 \%$ superior incompleto, 0,12\% superior incompleto, $19,94 \%$ relatou que não se aplica nenhuma das alternativas e $12,2 \%$ ignorou o questionamento.

A Tabela 3 relata sobre o tratamento do parceiro e esquema de tratamento que foi adotado pelo portados da Sífilis.

Tabela 3. Distribuição dos casos de gestantes com Sífilis quanto ao tratamento do parceiro e esquema de tratamento realizado.

\begin{tabular}{|c|c|c|c|c|c|c|c|}
\hline Variáveis & 2015 & 2016 & 2017 & 2018 & 2019 & Total & $\%$ \\
\hline \multicolumn{8}{|l|}{ Realizaçào de Tratamento } \\
\hline $\begin{array}{l}\text { Tratamento do parceiro } \\
\text { realizado }\end{array}$ & 45 & 63 & 78 & 147 & 51 & 384 & $35,4 \%$ \\
\hline $\begin{array}{l}\text { Tratamento do parceiro não } \\
\text { realizado }\end{array}$ & 173 & 132 & 113 & 63 & 24 & 505 & $57,7 \%$ \\
\hline $\begin{array}{l}\text { Tratamento do parceiro } \\
\text { ignorado }\end{array}$ & 6 & 9 & 14 & 15 & 5 & 49 & $6,9 \%$ \\
\hline \multicolumn{8}{|l|}{ Esquema de tratamento } \\
\hline Tratamento com Penicilina & 119 & 127 & 173 & 207 & 230 & 856 & $91,8 \%$ \\
\hline Outro esquema & 1 & 4 & 1 & - & 3 & 9 & $7,1 \%$ \\
\hline Não realizado & 5 & 8 & 8 & 31 & 15 & 67 & $1,5 \%$ \\
\hline Ignorado & 2 & - & 1 & 8 & 3 & 14 & $0,9 \%$ \\
\hline
\end{tabular}

Fonte: Pesquisa direta em base de dados. Teresina (2020). 
Foi observado que $35,4 \%$ realizou o tratamento, $57,7 \%$ não realizou tratamento e apenas $6,9 \%$ ignoraram o questionamento. No que se refere a esquema de tratamento, $91,8 \%$ informaram que fizeram o uso de Penicilina, 7,1\% informaram que utilizaram outro esquema de tratamento, $1,5 \%$ das gestantes não realizaram nenhum tratamento e $0,9 \%$ ignoraram o questionamento.

\section{Discussão}

Observou-se uma alta frequência de diagnósticos da sífilis em gestantes no terceiro trimestre de gravidez, podendo estar relacionada ao início tardio do pré-natal nas unidades básicas de saúde pelas gestantes e pela baixa qualidade da assistência realizada a essas gestantes durante as consultas de pré-natal. É fundamental uma detecção oportuna das gestantes com sífilis e a orientações sobre o tratamento correto das mesmas e dos seus parceiros sexuais (Cavalcante, Pereira \& Castro, 2017).

O VDRL é o exame mais utilizado durante o pré-natal para o rastreamento da sífilis gestacional, pois o mesmo apresenta alta especificidade e sensibilidade, podendo permanecer reagente mesmo após a cura. É indicado a realização do exame já na primeira consulta com a gestante, preferencialmente no primeiro trimestre da gravidez, o segundo exame na trigésima semana e o terceiro deve ser realizado na admissão hospitalar, seja por problemas na gestação e/ou assistência ao parto (de Oliveira \& Campos, 2020).

A não realização do pré-natal, ou a realização do mesmo, mas de forma inadequada, seja pelo início tardio ou por falta de comprometimento às consultas, também é um importante fator para o aumento significativo de sífilis gestacional. É de suma importância melhorar o acesso e a qualidade do pré-natal visando a promoção e prevenção das IST'S de forma geral. A baixa escolaridade e a faixa etária estão diretamente associadas à falta de conhecimento sobre a importância do pré-natal, tornando a adesão mais árdua (da Cunha, Monte, \& Nazaré, 2018)

É importante salientar que no presente estudo foi identificado um grande número de parceiros que não realizaram o tratamento contra sífilis junto com a gestante, que podem ser compreendidos por baixo entendimento da importância e procura para realização do pré-natal do parceiro justamente com a gestante, por fatores socioeconômicos, dificuldade de acesso à informação e aos serviços de saúde, e serviços inoperantes em localizar os parceiros e indicar o tratamento enfatizando a importância do mesmo ser concluído corretamente (Vasconcelos, 2017). 
O não tratamento do parceiro sexual da gestante infectada aumenta a probabilidade de uma transmissão da sífilis ao feto. É fundamental ressaltar a importância da realização do tratamento completo do parceiro e esclarecer qualquer duvidas que venham a surgir do casal por meio de educação em saúde para diminuir as chances de uma possível reinfecção e diminuir a incidência dos números de sífilis congênita (Monteiro \& Côrtes, 2019).

O estudo mostrou que $91,8 \%$ realizaram tratamento com a penicilina $\mathrm{G}$ benzatina, que é o medicamento de primeira escolha para o tratamento da sífilis em todos os estágios da doença e se mostra com altos níveis de eficácia desde que o esquema terapêutico seja realizado corretamente, o esquema é iniciado logo que é feito o diagnóstico da sífilis e é indicado tanto para a gestante quanto para o parceiro sexual ( Oliveira, Almeida, Marques, Silva \& Castro, 2020).

É importante salientar que menos depois do tratamento concluído, é necessário a realização de testes não treponêmicos nas gestantes durante o seu pré-natal mensalmente para o controle da cura. Para indicativo de sucesso no tratamento a resposta imunológica mais comum é a diminuição da titulação em duas diluições dos testes não treponemicos em até três meses e quatro diluições em até seis meses, com evolução até se obter o resultado de teste treponêmico não reagente. Mesmo depois de uma resposta imunológica adequada é indicado que prossiga com o seguimento clinico, com objetivo de monitorar possíveis reativação ou reinfecção (Conceição, Câmara \& Pereira, 2020).

A realização do pré-natal é necessária para prevenção e diagnósticos precoce de doenças e/ou de infecções sexualmente transmissíveis (ISTs). Foi revelado nesse estudo altas taxas de realização de pré-natal, mas uma assistência de qualidade, com captação precoce da gestante, com ações de promoção a saúde, orientação sexual e reprodutiva, realização do protocolo dos exames preconizados pelo ministério da saúde durante o período gestacional é essencial para a prevenção de danos ao bebe decorrente de possíveis consequências de uma sífilis gestacional (Padovani, Oliveira \& Pelloso, 2018).

Cabe ressaltar que os altos índices de baixa escolaridade é considerada um grande fator de risco para o surgimento da sífilis em gestantes, devido à falta de entendimento da população perante a importância das medidas de prevenção e promoção da saúde com essa população, além de incluir a realização de testes rápidos durantes os atendimento à mulher, bem como dos seus parceiros sexuais, independentemente do motivo da procura por atendimento nos serviços de saúde. Sabe- se que o uso de testes rápidos é de alto custoefetividade e de fácil execução, tornando-se uma importante ferramenta no diagnóstico e 
tratamento da sífilis precocemente das gestantes (Cardoso, Araújo, Cavalcante, Frota \& Melo, 2018).

A baixa escolaridade pode ser um fator limitante ao acesso as informações indispensáveis em relação ao autocuidado, a conscientização quanto a saúde sexual e medidas de prevenção contra doenças sexualmente transmissíveis (DST), como a sífilis. Diante disso, o desafio é garantir que gestantes infectadas tenham acesso ao tratamento qualificado da sífilis gestacional e prevenir uma possível complicação da doença (da Silva et al, 2019).

O presente estudo mostra que a prevalência dos casos de sífilis gestacional ocorre em jovens (15 a 19 anos) e adultos jovens (20 a 29 anos), o que ressalta que apesar do grande número de informações sobre as DSTs, a parcela mais jovem da população não se atenta à prevenção tornando-as mais susceptíveis ao contato. O início precoce da vida sexual pode ser considerado uma agravante frente as Dsts, o número elevado de gravidez na adolescência entre jovens, soma-se a ocorrência de doenças sexualmente transmissíveis, ajuda-nos a entender porque os jovens brasileiros são cada vez mais vulneráveis a infecções (Monteiro, Costa, Vieira \& Silva, 2015).

As gestantes com idade inferior a 20 anos, geralmente apresentam maior risco de contrair a infecção, devido a população adolescente estarem mais expostas as IST'S, em virtude de ser uma fase de imaturidade etária, cognitiva e emocional, além das influências sociais e descobertas. A relação sexual entre os adolescentes está cada vez mais precoce e com isso, a negligência no uso dos meios contraceptivos, o que se torna mais um fator para o aumento da gravidez na adolescência e as IST'S (Padovani, Rosseto, \& Marisa, 2018).

\section{Considerações Finais}

Os resultados desse estudo demonstram que há um grande caminho para percorrer, para alcançar a meta de eliminação de sífilis gestacional e congênita como um problema de saúde pública. A prevalência de Sífilis gestacional encontrada foi bastante elevada e as análises apontaram diversas variáveis relacionadas a sua ocorrência, tais como, ausência do pré-natal, esquema de tratamento inadequado, faixa etária, tratamento do parceiro, escolaridade e idade gestacional.

Para reduzir a prevalência de Sífilis gestacional e consequentemente reduzir a prevalência de Sífilis congênita é necessário que os profissionais de saúde e toda a comunidade saiba sobre a importância do diagnóstico precoce e do tratamento eficaz tanto da 
mulher quanto do seu parceiro. É essencial uma busca ativa para identificar as gestantes faltosas nas consultas, ações de conscientização de toda a população sobre as DSTs, enfatizando os riscos de ter práticas sexuais inseguras.

A detecção da Sífilis é feita por meio de testes rápidos que são disponibilizados gratuitamente pelo Ministério da Saúde, através do Sistema Único de Saúde (SUS), o que torna o teste acessível a todos os brasileiros. Todos devem estar atentos para evitar a infecção, pois coloca em risco a saúde tanto da mulher, do seu parceiro, quanto a do seu filho durante a gestação.

O artigo é de grande relevância para toda comunidade acadêmica e profissionais da área da saúde, uma vez que proporcionara mais informações atualizadas e com base cientifica acerca do tema para que haja melhores implementações de estratégias de intervenções e de maneira holística com essas mulheres durante sua gestação, visando a importância da realização do tratamento de maneira completa das gestantes e de seus parceiros sexuais, levando em consideração que a sífilis gestacional sem tratamento gera consequências significativas para o feto.

Com isso, sugere-se mais estudos em relação a esta temática para que se tenha mais conhecimento e qualificação dos profissionais de saúde a fim de prepara-los para prestar uma assistência de maneira mais holística e humanizada frente as gestantes com diagnóstico de sífilis gestacional durante as consultas de pré-natal objetivando a redução de possíveis complicações ao feto e a gestante em decorrência desta doença.

\section{Referências}

Brasil. (2019). Ministério da Saúde. Secretaria de Vigilância em Saúde. Departamento de Doenças de Condições Crônicas e Infecções Sexualmente Transmissíveis - DCCI. Boletim epidemiológico Sífilis 2019. Recuperado de http://www.aids.gov.br/pt-br/pub/2019/boletimepidemiologico-sifilis-2019

Cardoso, A. R. P., Araújo, M. A. L., Cavalcante, M. D. S., Frota, M. A., \& Melo, S. P. D. (2018). Análise dos casos de sífilis gestacional e congênita nos anos de 2008 a 2010 em Fortaleza, Ceará, Brasil. Ciência \& Saúde Coletiva, 23, 563-574.

Conceição, H. N. D., Câmara, J. T., \& Pereira, B. M. (2020). Análise epidemiológica e espacial dos casos de sífilis gestacional e congênita. Saúde em Debate, 43, 1145-1158. 
Cavalcante, P. A. D. M., Pereira, R. B. D. L., \& Castro, J. G. D. (2017). Sífilis gestacional e congênita em Palmas, Tocantins, 2007-2014. Epidemiologia e Serviços de Saúde, 26, 255264.

De Oliveira, E. H., de Almeida, A. T. A., Marques, M. C. A., da Silva, E. P., \& Castro, I. O. (2020). Impacto epidemiológico da Sífilis gestacional e congênita e sua relação com a mortalidade infantil no Estado do Piauí, Brasil. Research, Society and Development, 9(8), e856986539-e856986539.

da Silva, L. M. C., Dias, R. M., Frazão, A. D. G. F., da Silva Rezende, A. L., Moura, F. M. L., da Cunha Araújo, E., ... \& Leão, P. V. (2019). Sífilis congênita no estado do Pará-Brasil, 2007 a 2016. Revista Eletrônica Acervo Saúde, (24), e1003-e1003.

D'Oliveira, A. N. (2019). Sífilis em gestantes: qualidade dos dados e o perfil epidemiológico no estado de São Paulo (Doctoral dissertation, Universidade de São Paulo).

de Oliveira Campos, C., \& Campos, C. O. (2020). Abordagem diagnóstica e terapêutica da sífilis gestacional e congênita: revisão narrativa. Revista Eletrônica Acervo Saúde, (53), e3786-e3786.

Delben, T. V. T., \& Viana, T. R. (2018). Sífilis-Características e nova abordagem. Saúde \& Conhecimento-Jornal de Medicina Univag, 1.

FUNPEC. Lembre-se de se cuidar, sífilis: teste, trate e cure. Sífilis não. 13 de abril de 2017. Disponível em: https://www.sifilisnao.com.br/index_desktop.html\#. Acesso em: 10 de março de 2020.

Guerra, H. S., da Costa, C. V., dos Santos, I. A. B., da Silva, J. M., \& Barcelos, T. F. (2017). Sífilis congênita: repercussões e desafios. Arquivos Catarinenses de Medicina, 46(3), 194202.

Monteiro, M. D. O. P., Costa, M. C. O., Vieira, G. O., \& da Silva, C. A. L. (2015). Fatores associados à ocorrência de sífilis em adolescentes do sexo masculino, feminino e gestantes de 
um Centro de Referência Municipal/CRM-DST/HIV/AIDS de Feira de Santana, Bahia. Adolescencia e Saude, 12(3), 21-32.

Monteiro, R., \& de Resende Côrtes, P. P. (2019). A relação entre sífilis congênita e o tratamento do parceiro da gestante: um estudo epidemiológico. Revista PróUniverSUS, 10(2), 13-17.

Padovani, C., Oliveira, R. R. D., \& Pelloso, S. M. (2018). Sífilis na gestação: associação das características maternas e perinatais em região do sul do Brasil. Revista Latino-Americana de Enfermagem, 26.

Patias, N. D., \& Hohendorff, J. V. (2019). Critérios de qualidade para artigos de pesquisa qualitativa. Psicologia em estudo, 24.

Pereira, A. S., Shitsuka, D. M., Parreira, F.J., Shitsuka, R. (2018). Metodologia da pesquisa cientifica. 1.ed - santa maria, RS: UFSM, NTE, 69.

Ros-Vivancos, C., González-Hernández, M., Navarro-Gracia, J. F., Sánchez-Payá, J., González-Torga, A., \& Portilla-Sogorb, J. (2018). Evolución del tratamiento de la sífilis a lo largo de la historia. Revista Española de Quimioterapia, 31(6), 485.

Sobral, C. (2012). Diagnóstico e tratamento da sífilis: uma investigação com mulheres assistidas na atenção básica em Sobral, Ceará.rESUMoS dE diSSErtaçõES E tESES/ABSTRACTS OF THESIS, 24(4), 239-245.

Torres, R. G., Mendonça, A. L. N., Montes, G. C., Manzan, J. J., Ribeiro, J. U., \& Paschoini, M. C. (2019). Sífilis na gestação: a realidade em um hospital público. Rev Bras Ginecol Obstet, 90-96.

Vasconcelos, M. I. O., de Oliveira, K. M. C., Magalhães, A. H. R., Guimarães, R. X., Linhares, M. D. S. C., de Oliveira Queiroz, M. V., \& Albuquerque, I. M. A. N. (2017). Sífilis na gestação: estratégias e desafios dos enfermeiros da atenção básica para o tratamento simultâneo do casal. Revista Brasileira em Promoção da Saúde, 29, 85-92. 
Research, Society and Development, v. 9, n. 10, e8889109414, 2020

(CC BY 4.0) | ISSN 2525-3409 | DOI: http://dx.doi.org/10.33448/rsd-v9i10.9414

\section{Porcentagem de contribuição de cada autor no manuscrito}

Francisca Flaviana Alves Sobrinho - 40\%

Sara Maria Marques Lima - 40 \%

Tatiana Maria Melo Guimarães - 20\% 